11,01

\title{
Влияние наноразмерных эффектов на состав сосуществующих фаз в бинарной системе с искривленными границами
}

\author{
(C) М.А. Шебзухова, А.А. Шебзухов \\ Кабардино-Балкарский государственный университет им. Х.М. Бербекова, \\ Нальчик, Россия \\ E-mail: sh-madina@mail.ru
}

(Поступила в Редакцию 10 ноября 2016 г.

В окончательной редакции 15 декабря 2016 г.)

В рамках термодинамики фазовых равновесий с учетом поверхностных явлений получены новые соотношения в интегральной форме для бинарных систем в случае, когда одна из фаз диспергирована (до наноразмеров) внутри другой (матрицы). Полученные соотношения использованы для построения размернозависимой фазовой диаграммы состояния в бинарной системе $\mathrm{Mo}-\mathrm{Ru}$, содержащей твердые наночастицы с ОЦК- и ГПУ-структурами и жидкую фазу в условии равновесия. При проведении расчетов последовательно учтены размерные зависимости характеристик чистых компонентов и параметра межчастичного взаимодействия в фазах. Для макроскопического случая результаты расчета согласуются с экспериментальными данными.

DOI: $10.21883 /$ FTT.2017.07.44602.410

\section{1. Введение}

Искривление поверхности, находящейся между сосуществующими фазами, влияет на основные параметры термодинамического равновесия в системе по сравнению со случаем равновесия плоского слоя с окружающими фазами [1]. За последнее время актуальность приобрели вопросы влияния искривления поверхности на характеристики самих фаз, сосуществующих в равновесных условиях. Особое внимание при этом привлекают ситуации, когда речь идет о размерах кривизны в наноразмерном диапазоне. Такой интерес, в частности, связан с эффектами по влиянию нановыделений (нанопреципитатов) на важнейшие эксплутационные характеристики промышленно значимых сплавов, в том числе на жаропрочность, механические свойства и радиационную стойкость материалов, используемых в атомной технике [2,3]. Бинарные сплавы $\mathrm{Fe}-\mathrm{Cr}$ и многокомпонентные сплавы на их основе, которые используются широко для разработки перспективных типов стали, в том числе европейской стали типа EUROFER, характеризуются наличием наноразмерных фазовых выделений $[4,5]$.

Решение подобных задач по установлению влияния наноразмерных эффектов на свойства гетерогенных систем на данном этапе сопряжено с большими трудностями. По этой причине во многих работах используют упрощенные подходы, связанные с теми или иными предположениями, в том числе при учете межфазовой сегрегации компонентов, размерной зависимости межфазного натяжения, температуры равновесия, скачка энтропии при фазовом переходе и ряда других параметров (см. например, [6]).

По нашему мнению, на данном этапе надежным фундаментом для установления влияния искривления на основные параметры термодинамического равновесия является классическая термодинамика фазовых равновесий в системах с искривленными границами при строгом учете поверхностных явлений [1,7-9].

Целью настоящей работы является получение соотношений для определения состава фаз, расположенных по разные стороны от искривленного поверхностного слоя в бинарной системе в условиях равновесия в рамках термодинамической теории фазовых равновесий с учетом поверхностных явлений и проведение численных расчетов для бинарной системы Mo-Ru.

\section{2. Основные соотношения, связывающие состав, температуру и степень дисперсности в бинарной системе}

Рассмотрим бинарную систему, состоящую из двух фаз $(\alpha, \beta)$ и искривленного поверхностного (межфазного) слоя сферической формы между ними $(\sigma)$, находящуюся в равновесном состоянии. Выберем в качестве переменных молярные доли компонентов в фазах $x_{i}^{(\alpha)}, x_{i}^{(\beta)}$, в межфазном слое $x_{i}^{(\sigma)}$, температуру $T$, давления $P^{(\alpha)}$ и $P^{(\beta)}$, а также радиус $r$, который является характеристикой степени дисперсности. В качестве разделяющей поверхности выберем поверхность натяжения Гиббса [1]. Будем считать, что фазы $\alpha$ и $\beta$ располагаются соответственно с вогнутой и выпуклой стороны от поверхности натяжения. В ходе рассуждений будем использовать еще одну разделяющую поверхность с радиусом $r_{e}$, называемую эквимолекулярной разделяющей поверхностью [1] и с ее использованием введем в рассмотрение параметр Толмена $\delta=r_{e}-r$. 
Для равновесного состояния термодинамический потенциал $g$ двухфазной $(\alpha, \beta)$ системы имеет глобальный минимум. При этом должны выполняться соотношения

$$
\begin{gathered}
\frac{\partial g^{(\alpha)}}{\partial x_{i}^{(\alpha)}}=\frac{\partial g^{(\beta)}}{\partial x_{i}^{(\beta)}}, \\
g^{(\alpha)}-x_{i}^{(\alpha)} \frac{\partial g^{(\alpha)}}{\partial x_{i}^{(\alpha)}}=g^{(\beta)}-x_{i}^{(\beta)} \frac{\partial g^{(\beta)}}{\partial x_{i}^{(\beta)}},
\end{gathered}
$$

где $g^{(\xi)}$ - термодинамический потенциал фазы $\xi$ (на моль), $x_{i}^{(\xi)}$ - молярная концентрация $i$-компонента в фазе $\xi(\xi=\alpha, \beta, i=1,2)$.

Давления в сосуществующих фазах связаны соотношением Кондо [1]

$$
P^{(\alpha)}-P^{(\beta)}=\frac{2 \sigma_{x}}{r_{x}}+\left(\frac{d \sigma_{x}}{d r_{x}}\right)^{*},
$$

где звездочка указывает, что производная относится к мысленному перемещению разделяющей поверхности (не к реальному физическому процессу). При выборе в качестве разделяющей поверхности, поверхности натяжения $\left(\frac{d \sigma_{x}}{d r_{x}}\right)=0$ будем иметь

$$
d P^{(\alpha)}=d P^{(\beta)}+\frac{2}{r} d \sigma-\frac{2 \sigma}{r^{2}} d r .
$$

Для двухкомпонентной двухфазной системы с искривленной границей, находящейся в равновесном состоянии, используя (1) и (2) с учетом (4), можно получить следующие соотношения

$$
\begin{aligned}
\omega_{i} d \sigma= & -\left(s_{i}^{(\sigma)}-s_{i}^{(\alpha)}\right) d T+\left(v_{i \alpha}^{(\sigma)}-v_{i}^{(\alpha)}\right) d P^{(\alpha)} \\
& +v_{i \beta}^{(\sigma)} d P^{(\beta)}-x_{j}^{(\sigma)} g_{j j}^{(\sigma)} d x_{j}^{(\sigma)}+x_{j}^{(\alpha)} g_{j j}^{(\alpha)} d x_{j}^{(\alpha)}, \\
\left(s_{i}^{(\beta)}-s_{i}^{(\alpha)}\right) d T+ & v_{i}^{(\alpha)} d P^{(\alpha)}-v_{i}^{(\beta)} d P^{(\beta)} \\
= & x_{j}^{(\alpha)} g_{j j}^{(\alpha)} d x_{j}^{(\alpha)}-x_{j}^{(\beta)} g_{j j}^{(\beta)} d x_{j}^{(\beta)} .
\end{aligned}
$$

В этих выражениях $\sigma$ - межфазное (поверхностное) натяжение на границе фаз $\alpha$ и $\beta$, отнесенное к поверхности натяжения, $s_{i}, v_{i}, \omega_{i}$ - парциальные значения энтропии, объема и поверхности в расчете на моль для $i$-компонента соответственно, $v_{i \alpha}^{(\sigma)}$ и $v_{i \beta}^{(\sigma)}-$ парциальные значения взаимно дополняющих друг друга частей среднего молярного объема поверхностного слоя для $i$-компонента $v_{i}^{(\sigma)}$, расположенные соответственно со стороны $\alpha$ - и $\beta$-фаз, $g_{j j} \equiv \frac{\partial^{2} g}{\left(\partial x_{j}\right)^{2}}$.

Для описания фазового равновесия двух конденсированных фаз бинарной системы в макроскопическом случае $(r=\infty)$ довольно часто используется условие постоянства давления $(P=$ const $)$ и находятся уравнения, выражающие зависимость состава фаз от температуры. Таким образом теоретически строятся фазовые диаграммы состояния с линиями ликвидуса и солидуса $[10,11]$.
Обратимся к случаю, когда фиксируется давление в фазе $\beta\left(P^{(\beta)}=\mathrm{const}\right)$. Описание равновесия фаз проведем для бинарной системы с заданной степенью дисперсности (с постоянным, но конечным радиусом кривизны).

В связи с трудностями нахождения статистической суммы раствора воспользуемся приближением ближайших соседей и введем в рассмотрение энергетический параметр, характеризующий взаимодействие частиц (энергия смешения) $Q=z N_{0}\left(2 \varepsilon_{i j}-\varepsilon_{i i}-\varepsilon_{j j}\right) / 2$, где $\varepsilon_{i j}, \varepsilon_{i i}, \varepsilon_{j j}-$ средние значения энергии парного взаимодействия соответствующих частиц, $z$ - координационное число, $N_{0}$ - число Авогадро. Для поэтапного описания термодинамических свойств фаз обратимся к разложению логарифмов коэффициентов активности компонентов $f_{i}$ в ряд по степеням $\xi=Q / R T$. Если ограничиться первым членом разложения $\left(\left(\ln f_{i}\right)_{\xi=0}\right)$ будем иметь (идеальный раствор) $g_{j j}=R T /\left(x_{i} x_{j}\right)$. При удержании в этом разложении члена с первой степенью $\xi$ (нулевое приближение теории регулярных растворов), получаем $g_{j j}=R T\left[\left(x_{i} x_{j}\right)^{-1}-2 Q / R T\right]$ (напомним, что $\left.x_{i}+x_{j}=1\right)$. Вычисление этой производной в следующем приближении не представляет трудностей.

В случае перехода к однокомпонентной системе $\left(x_{i}^{(\alpha)}=x_{i}^{(\beta)}=x_{i}^{(\sigma)}=1, x_{j}^{(\alpha)}=x_{j}^{(\beta)}=x_{j}^{(\sigma)}=0\right)$ из (4), (5) и (6) получаются соотношения, описывающие соответствующие размерные эффекты, в том числе зависимости поверхностного (межфазного) натяжения и температуры фазового равновесия (например, температуры плавления) от размера $[12,13]$.

Из исходных соотношений (4) и (6) в рамках модели раствора, использованной в [3] (соответствует случаю учета слагаемого $\left(\frac{\partial \ln f_{i}}{\partial \xi}\right)_{\xi=0} \cdot \xi$ в разложении $\ln f_{i}$ по степеням $\xi$ ), будем иметь

$$
\begin{aligned}
\left(s_{0 i}^{(\beta)}\right. & \left.-s_{0 i}^{(\alpha)}\right) d T+\frac{2 v_{0 i}^{(\alpha)}}{r} d \sigma=D\left(R T \ln x_{i}^{(\beta)} / x_{i}^{(\alpha)}\right) \\
& +2 Q^{(\alpha)}\left(1-x_{i}^{(\alpha)}\right) d x_{i}^{(\alpha)}-2 Q^{(\beta)}\left(1-x_{i}^{(\beta)}\right) d x_{i}^{(\beta)}
\end{aligned}
$$

где нижний индекс 0 указывает на принадлежность соответствующей величины к чистым компонентам. В первом слагаемом правой части этого выражения для обозначения дифференциала по составу при неизменных параметрах состояния, кроме состава, следуя Гиббсу, мы использовали букву $D$.

В термодинамике фазовых равновесий в бинарных и многокомпонентных системах для температур, выше температуры Дебая, в том числе в расчетах, основанных на подходе GALPHAD [6], допускается независимость от температуры скачка энтропии $\Delta s_{0 i}^{(\alpha \rightarrow \beta)}=s_{0 i}^{(\beta)}-s_{0 i}^{(\alpha)}$ при $\alpha \rightarrow \beta$ фазовом переходе. Воспользуемся этим допущением и для конденсированных фаз вдали от критических условий предположим независимость параметра квазихимического взаимодействия $Q$ от давления, что уже использовано во втором слагаемом в левой части (7). Отметим, что при этих допущениях сохраняются раз- 
мерные зависимости энтропии, объема и поверхности чистых компонентов.

С учетом изложенного выше будем иметь первую систему из двух уравнений

$$
\begin{aligned}
& \frac{2 v_{0 i}^{(\alpha)}(r)}{r}\left[\sigma-\sigma_{0 i}\left(r, T_{0 i}\right)\right]=\left[s_{0 i}^{(\beta)}(r)-s_{0 i}^{(\alpha)}(r)\right]\left[T_{0 i}(r)-T\right] \\
& +R T \ln \frac{x_{i}^{(\beta)}}{x_{i}^{(\alpha)}}+Q^{(\beta)}(r)\left(1-x_{i}^{(\beta)}\right)^{2}-Q^{(\alpha)}(r)\left(1-x_{i}^{(\alpha)}\right)^{2},
\end{aligned}
$$$$
\frac{2 v_{0 j}^{(\alpha)}(r)}{r}\left[\sigma-\sigma_{0 j}\left(r, T_{0 j}\right)\right]=\left[s_{0 j}^{(\beta)}(r)-s_{0 j}^{(\alpha)}(r)\right]\left[T_{0 j}(r)-T\right]
$$$$
+R T \ln \frac{1-x_{i}^{(\beta)}}{1-x_{i}^{(\alpha)}}+Q^{(\beta)}(r)\left(x_{i}^{(\beta)}\right)^{2}-Q^{(\alpha)}(r)\left(x_{i}^{(\alpha)}\right)^{2},
$$

где $T_{0 i}(r), T_{0 j}$ и $\sigma_{0 i}\left(r, T_{0 i}\right), \sigma_{0 j}\left(r, T_{0 j}\right)$ - температуры равновесия $\alpha$ - и $\beta$-фаз для компонентов и межфазные натяжения компонентов $i$ и $j$ при этих температурах, которые зависят от размера.

Уравнения (8) и (9) для межфазного натяжения на границе $\alpha$ и $\beta$ фаз не имеют аналогов для плоских поверхностей и при $r \rightarrow \infty$, они переходят в соотношения, связывающие состав сосуществующих фаз с температурой в макроскопической системе в рамках теории регулярных растворов (уравнения линий ликвидуса и солидуса) $[10,11]$. Если дополнительно (к условию $P^{(\beta)}=$ const) зафиксировать температуру и принять независимость межфазного натяжения и молярного объема от размера в приближении идеальных растворов, из исходных соотношений аналогичным образом можно получить известную формулу Оствальда-Фройндлиха по растворимости малых кристаллов [14].

Следующую систему из двух уравнений можно получить с использованием исходных соотношений (5), (6) и (4). Предварительно преобразуем первые сомножители в первых двух слагаемых в правой части соотношения (5). Воспользуемся очевидным соотношением $v_{i}^{(\sigma)}=v_{0 i}^{(\sigma)}=v_{0 i \alpha}^{(\sigma)}+v_{0 i \beta}^{(\sigma)}$ и учтем, что второе слагаемое в случае, когда поверхность имеет сферическую форму, выражается в виде [12]

$$
v_{0 i \beta}^{(\sigma)}=\frac{v_{0 i}^{(\sigma)}-v_{0 i}^{(\alpha)}}{v_{0 i}^{(\beta)}-v_{0 i}^{(\alpha)}} v_{0 i}^{(\beta)}+\omega_{0 i} \delta_{0 i}\left(1+\frac{\delta_{0 i}}{r}+\frac{1}{3} \frac{\delta_{0 i}^{2}}{r^{2}}\right),
$$

где $\delta_{0 i}-$ параметр Толмена для $i$-компонента. Из (5), (6) и (4) следует для однокомпонентной системы

$$
\begin{aligned}
s_{0 i}^{(\sigma)} & -s_{0 i}^{(\alpha)}=-\omega_{0 i}\left(\frac{d \sigma_{0 i}}{d T}\right)_{P^{(\beta)}}+\left(s_{0 i}^{(\beta)}-s_{0 i}^{(\alpha)}\right) \\
& \times\left[\frac{v_{0 i}^{(\sigma)}-v_{0 i}^{(\alpha)}}{v_{0 i}^{(\beta)}-v_{0 i}^{(\alpha)}}+\frac{\omega_{0 i} \delta_{0 i}}{v_{0 i}^{(\alpha)}}\left(1+\frac{\delta_{0 i}}{r}+\frac{1}{3} \frac{\delta_{0 i}^{2}}{r^{2}}\right)\right] .
\end{aligned}
$$

В макроскопической системе, как показано в [1], учет условий равновесия плоского поверхностного слоя с прилегающими фазами равносилен выбору эквимолекулярной разделяющей поверхности $\left(r=r_{e}\right)$. В таком случае $\delta=r_{e}-r=r_{e}-r_{e}=0$ и в квадратных скобках в (11) отличным от нуля остается первое слагаемое. При этом из (11) следует выражение для температурного коэффициента поверхностного (межфазного) натяжения в макроскопическом случае

$$
\omega_{0 i \infty} \frac{d \sigma_{0 i \infty}}{d T}=\left(s_{0 i \infty}^{(\beta)}-{ }_{0 i \infty}^{(\alpha)}\right) \frac{v_{0 i \infty}^{(\sigma)}-v_{0 i \infty}^{(\alpha)}}{v_{0 i \infty}^{(\beta)}-v_{0 i \infty}^{(\alpha)}}-\left(s_{0 i \infty}^{(\sigma)}-s_{0 i \infty}^{(\alpha)}\right),
$$

которое было получено в [1] (индекс „ळ“ указывает на условие $r=\infty$ ).

С учетом изложенного выше (в случае разложения $\ln f_{i}$ до члена с первой степенью $\left.\xi=Q / R T\right)$, будем иметь

$$
\begin{aligned}
\omega_{0 i} d \sigma= & D\left\{R T \ln \left[K_{i}^{(\sigma \alpha)} / K_{i}^{(\beta \alpha)^{\bar{\rho}_{0 i}}}\right]\right\}+2\left[Q^{(\sigma)} x_{j}^{(\sigma)} d x_{j}^{(\sigma)}\right. \\
& \left.-\left(1-\bar{\rho}_{0 i}\right) Q^{(\alpha)} x_{j}^{(\alpha)} d x_{j}^{(\alpha)}-Q^{(\beta)} x_{j}^{(\beta)} d x_{j}^{(\beta)}\right], \quad(13)
\end{aligned}
$$

где $K_{i}^{(\sigma \alpha)}=x_{i}^{(\sigma)} / x_{i}^{(\alpha)}, K_{i}^{(\beta \alpha)}=x_{i}^{(\beta)} / x_{i}^{(\alpha)}, K_{i}^{(\sigma \alpha)}, K_{i}^{(\beta \alpha)}-$ коэффициенты распределения $i$-компонента между сосуществующими фазами,

$$
\bar{\rho}_{0 i}=\frac{v_{0 i}^{(\sigma)}-v_{0 i}^{(\alpha)}}{v_{0 i}^{(\beta)}-v_{0 i}^{(\alpha)}}+\frac{\omega_{0 i} \delta_{0 i}}{v_{0 i}^{(\alpha)}}\left(1+\frac{\delta_{0 i}}{r}+\frac{1}{3} \frac{\delta_{0 i}^{2}}{r^{2}}\right),
$$

Буква $D$ в первом слагаемом в правой части (13) указывает на постоянство температуры при дифференцировании. Соотношение, аналогичное (13), можно записать через характеристики другого компонента. Интегрирование этих двух выражений при изменении концентраций от единицы до текущих значений $x_{i}^{(\alpha)}, x_{i}^{(\beta)}, x_{i}^{(\sigma)}$ для систем с заданной степенью дисперсности позволяет получить следующие уравнения

$$
\begin{aligned}
\sigma & =\sigma_{0 i}(T, r)+\frac{R T}{\omega_{0 i}(r)}\left\{\ln \frac{x_{i}^{(\sigma)}}{x_{i}^{(\alpha)}}-\bar{\rho}_{0 i}(r) \ln \frac{x_{i}^{(\beta)}}{x_{i}^{(\alpha)}}\right. \\
+ & \frac{Q^{(\sigma)}(r)}{R T}\left(1-x_{i}^{(\sigma)}\right)^{2}-\left[1-\bar{\rho}_{0 i}(r)\right] \frac{Q^{(\alpha)}(r)}{R T}\left(1-x_{i}^{(\alpha)}\right)^{2} \\
- & \left.\bar{\rho}_{0 i}(r) \frac{Q^{(\beta)}(r)}{R T}\left(1-x_{i}^{(\beta)}\right)^{2}\right\}, \\
\sigma & =\sigma_{0 j}(T, r)+\frac{R T}{\omega_{0 j}(r)}\left\{\ln \frac{x_{j}^{(\sigma)}}{x_{j}^{(\alpha)}}-\bar{\rho}_{0 j}(r) \ln \frac{x_{j}^{(\beta)}}{x_{j}^{(\alpha)}}\right. \\
& +\frac{Q^{(\sigma)}(r)}{R T}\left(x_{i}^{(\sigma)}\right)^{2}-\left[1-\bar{\rho}_{0 j}(r)\right] \frac{Q^{(\alpha)}(r)}{R T}\left(x_{i}^{(\alpha)}\right)^{2} \\
& \left.-\bar{\rho}_{0 j}(r) \frac{Q^{(\beta)}(r)}{R T}\left(x_{i}^{(\beta)}\right)^{2}\right\},
\end{aligned}
$$


где

$$
\begin{gathered}
\sigma_{0 i}(r, T)=\sigma_{0 i}\left(r, T_{0 i}\right)+\left(\frac{d \sigma_{0 i}}{d T}\right)_{P^{(\beta)}} \cdot\left[T-T_{0 i}(r)\right], \\
\sigma_{0 j}(r, T)=\sigma_{0 j}\left(r, T_{0 j}\right)+\left(\frac{d \sigma_{0 j}}{d T}\right)_{P^{(\beta)}} \cdot\left[T-T_{0 j}(r)\right],
\end{gathered}
$$

$\bar{\rho}_{0 j}$ выражается также как (14) с заменой индекса $i$ на $j$.

При $r \rightarrow \infty$ из (15) получаем следующее уравнение изобары межфазного натяжения в бинарной макроскопической системе в рамках термодинамики регулярных растворов:

$$
\begin{aligned}
\sigma_{\infty}= & \sigma_{0 i \infty}(T)+\frac{R T}{\omega_{0 i \infty}}\left[\frac{x_{i \infty}^{(\sigma)}}{x_{i \infty}^{(\alpha)}}\left(\frac{x_{i \infty}^{(\alpha)}}{x_{i \infty}^{(\beta)}}\right)_{\rho^{0 i \infty}}\right] \\
& +\frac{Q_{\infty}^{(\sigma)}}{\omega_{0 i \infty}}\left(1-x_{i}^{(\sigma)}\right)^{2}-\left(1-\rho_{0 i \infty}\right) \frac{Q_{\infty}^{(\alpha)}}{\omega_{0 i \infty}}\left(1-x_{i}^{(\alpha)}\right)^{2} \\
& -\rho_{0 i \infty} \frac{Q_{\infty}^{(\beta)}}{\omega_{0 i \infty}}\left(1-x_{i}^{(\beta)}\right)^{2},
\end{aligned}
$$

где $\quad \sigma_{0 i \infty}(T)=\sigma_{0 i \infty}\left(T_{0 i \infty}\right)+\frac{d \sigma_{0 i \infty}}{d T}\left(T-T_{0 i \infty}\right), \quad \rho_{0 i \infty}=$ $=\left(v_{0 i \infty}^{(\sigma)}-v_{0 i \infty}^{(\alpha)}\right) /\left(v_{0 i \infty}^{(\beta)}-v_{0 i \infty}^{(\alpha)}\right)$.

Уравнения (19) внешне совпадает с уравнением изотермы межфазного натяжения на границе двух растворов [15], но необходимо иметь в виду, что в (19) фигурируют сопряженные значения температуры и состава сосуществующих фаз.

Для бинарной системы жидкость $(\alpha)-$ пар $(\beta)$, в силу того, что $v_{0 i \infty}^{(\sigma)} \ll v_{0 i \infty}^{(\beta)} \gg v_{0 i \infty}^{(\alpha)}$ имеем $\rho_{0 i \infty} \approx 0$ и (19) переходит в уравнение

$$
\begin{aligned}
\sigma_{\infty}= & \sigma_{0 i \infty}(T)+\frac{R T}{\omega_{0 i \infty}} \ln \frac{x_{i}^{(\sigma)}}{x_{i}^{(\alpha)}}+\frac{Q_{\infty}^{(\sigma)}}{\omega_{0 i \infty}}\left(1-x_{i \infty}^{(\sigma)}\right)^{2} \\
& -\frac{Q_{\infty}^{(\alpha)}}{\omega_{0 i \infty}}\left(1-x_{i \infty}^{(\alpha)}\right)^{2},
\end{aligned}
$$

которое совпадает с уравнением Батлера-Жуховицкого для поверхностного натяжения раствора, подчиняющегося нулевому приближению теории регулярных растворов [16].

Система четырех уравнений (8), (9) и (15), (16) позволяет определить состав сосуществующих фаз $x_{i}^{(\alpha)}, x_{i}^{(\beta)}$, межфазного слоя $x_{i}^{(\sigma)}$ и межфазное натяжение $\sigma$ при разных температурах для заданного значения радиуса поверхности натяжения $r$. Настоящая работа содержит результаты расчетов для состава сосуществующих фаз, при нахождении которых на промежуточных этапах приходится рассчитывать значения $x_{i}^{(\sigma)}$ и $\sigma$.

Отметим, что из соотношений (5), (6) и (4) наряду с (15) и (16) можно получить следующую (вторую) систему из двух уравнений в рамках той же модели растворов:

$$
\begin{aligned}
\sigma & =\sigma_{0 i}(T, r)+\frac{R T}{\omega_{0 i}(r)}\left[1+\frac{2 v_{0 i}^{(\alpha)}(r)}{r \omega_{0 i}^{(\alpha)}(r)} \bar{\rho}_{0 i}(r)\right]^{-1} \\
\times & {\left[\ln \frac{x_{i}^{(\sigma)}}{x_{i}^{(\alpha)}}+\left(1-x_{i}^{(\sigma)}\right)^{2} \frac{Q^{(\sigma)}(r)}{R T}-\left(1-x_{i}^{(\alpha)}\right)^{2} \frac{Q^{(\alpha)}(r)}{R T}\right], } \\
\sigma= & \sigma_{0 j}(T, r)+\frac{R T}{\omega_{0 j}(r)}\left[1+\frac{2 v_{0 j}^{(\alpha)}(r)}{r \omega_{0 j}^{(\alpha)}(r)} \bar{\rho}_{0 j}(r)\right]^{-1} \\
& \times\left[\ln \frac{1-x_{j}^{(\sigma)}}{1-x_{j}^{(\alpha)}}+\left(x_{j}^{(\sigma)}\right)^{2} \frac{Q^{(\sigma)}(r)}{R T}-\left(x_{j}^{(\alpha)}\right)^{2} \frac{Q^{(\alpha)}(r)}{R T}\right] .
\end{aligned}
$$

В заключение этого раздела приведем уравнения, которые следуют из исходных соотношений при использовании уравнения Гиббса-Дюгема. В силу того что вторые производные термодинамического потенциала по составу берутся при постоянстве температуры и давления, будем иметь

$$
\begin{aligned}
\sigma= & \sigma_{0 v}(T, r)+\frac{R T}{\omega_{0 v}(r)} \\
& \times \ln \left[\frac{\alpha_{v}^{(\sigma)}(r)}{\left(\alpha_{v}^{(\alpha)}(r)\right)\left(1-\bar{\rho}_{0 v}(r)\right)\left(\alpha_{v}^{(\beta)}(r)\right)\left(\bar{\rho}_{0 v}(r)\right)}\right], \\
\sigma= & \sigma_{0 v}(T, r)+\frac{R T}{\omega_{0 v}(r)} \\
& \times\left[1+\frac{2 v_{0 v}^{(\alpha)}(r)}{r \omega_{0 v}(r)} \bar{\rho}_{0 v}(r)\right]^{-1} \ln \left[\frac{\alpha_{v}^{(\sigma)}(r)}{\alpha_{v}^{(\alpha)}(r)}\right],
\end{aligned}
$$

где $a_{v}$ - термодинамическая активность компонента $v$ $(v=i, j)$, которая зависит наряду с другими параметрами состояния и от радиуса поверхности натяжения дисперсной частицы.

\section{3. Размерные зависимости индивидуальных характеристик компонентов и параметра квазихимического взаимодействия частиц в растворе}

3.1. Зависимость межфазного натяжения и температуры равновесия от радиуса поверхности натяжения дисперсной частицы, находящейся в матрице, в однокомпонентной системе в изобарических усл о в и я х. Для проведения численных расчетов по приведенным выше соотношениям необходимо располагать зависимостями межфазного натяжения $\sigma_{0 i}$ и температуры 
равновесия $\alpha$ - и $\beta$-фаз $T_{0 i}$ компонентов от размера $r$ при постоянстве давления в фазе $\beta$. Для установления таких зависимостей обратимся к исходным соотношениям (2) и (4). Из этих выражений следует дифференциальное уравнение

$$
\frac{1}{\sigma_{0 i}}\left(\frac{d \sigma_{0 i}}{d r}\right)_{P^{(\beta)}}=\frac{\frac{2}{r^{2}}\left\{\delta_{0 i}\left(1+\frac{\delta_{0 i}}{r}+\frac{1}{3} \frac{\delta_{0 i}^{2}}{r^{2}}\right)+\delta_{0 i}^{*}\right\}}{1+\frac{2}{r}\left\{\delta_{0 i}\left(1+\frac{\delta_{0 i}}{r}+\frac{1}{3} \frac{\delta_{0 i}^{2}}{r^{2}}\right)+\delta_{0 i}^{*}\right\}},
$$

где

$$
\delta_{0 i}^{*}=\frac{v_{0 i}^{(\alpha)}}{\omega_{0 i}}\left(\frac{v_{0 i}^{(\sigma)}-v_{0 i}^{(\alpha)}}{v_{0 i}^{(\beta)}-v_{0 i}^{(\alpha)}}-\frac{s_{0 i}^{(\sigma)}-s_{0 i}^{(\alpha)}}{s_{0 i}^{(\beta)}-s_{0 i}^{(\alpha)}}\right) .
$$

Соотношение (25) получено нами [17], оно является аналогом уравнения Гиббса-Толмена-Кенига-Баффа (ГТКБ) при условии постоянства давления во внешней фазе $P^{(\beta)}=$ const.

При нахождении решения уравнения ГТКБ, справедливого при $T=$ const, как правило, параметр Толмена $\delta_{0 i}$ считается не зависящим от $r$ (см., например, $[1,17]$ ). При таком предположении из полученного решения (в случае малых искривлений поверхности) следует формула Толмена.

Сохраним условие $\left(\delta_{0 i}=\mathrm{const}\right)$ и дополнительно будем считать не зависящей от $r$ величину $\delta_{0 i}^{*}\left(\delta_{0 i}^{*}=\right.$ const $)$. При таких предположениях из (25) можно получить, как показано в [17],

$$
\sigma_{0 i}(r)=A_{0 i} \sigma_{0 i \infty} r \frac{\exp \left[D_{0 i} \operatorname{arctg}\left(\frac{2 r+b_{0 i} \delta_{0 i}}{\delta_{0 i} D_{0 i}}\right)\right]}{\left(r+a_{0 i} \delta_{0 i}\right)^{m_{0 i}}\left(r^{2}+b_{0 i} \delta_{0 i} r+c_{0 i} \delta_{0 i}^{2}\right)^{n_{0 i}}},
$$

где $\quad A_{0 i}=\exp \left[-\sqrt{4 c_{0}-b_{0}^{2}} \operatorname{arctg}(\infty)\right], \quad b_{0}=1.4425$, $c_{0}=1.1958, \quad D_{0 i}=\sqrt{4 c_{0 i}-b_{0 i}^{2}}, \quad m_{0 i}=1-a_{0 i}, \quad n_{0 i}=$ $=\left(1-m_{0 i}\right) / 2$. Значения $a_{0 i}, b_{0 i} \quad$ и $c_{0 i}$ зависят от $d_{0 i}=1+\delta_{0 i}^{*} / \delta_{0 i}$ и находятся из совместного решения уравнений $a_{0 i}+b_{0 i}=2 d_{0 i}, c_{0 i}+a_{0 i} b_{0 i}=2, a_{0 i} c_{0 i}=2 / 3$.

Из системы уравнений (5), (6) и (4) при тех же предположениях можно получить выражение для размерной зависимости температуры фазового равновесия [18]

$$
\begin{aligned}
T_{0 i}(r)= & T_{0 i \infty}-A_{0 i} \frac{2 \sigma_{0 i \infty} v_{0 i \infty}^{(\alpha)}}{\left(s_{0 i \infty}^{(\beta)}-s_{0 i \infty}^{(\alpha)}\right)} \\
& \times \frac{\exp \left[D_{0 i} \operatorname{arctg}\left(\frac{2 r+b_{0 i} \delta_{0 i}}{\delta_{0 i} D_{0 i}}\right)\right]}{\left(r+a_{0 i} \delta_{0 i}\right)^{\left(1-3 a_{0 i} / 2\right)}\left(r^{3}+2 d_{0 i} \delta_{0 i} r^{2}\right)}
\end{aligned}
$$

При больших значениях радиуса поверхности натяжения из (28) следует формула Томсона. Результаты расчетов с использованием (28), как показано в [18], достаточно хорошо согласуются с имеющимися экспериментальными данными.
3.2. Размерные зависимости $v_{0 i}, \quad \omega_{0 i}$ и $\Delta s_{0 i}^{(\alpha \rightarrow \beta)}=s_{0 i}^{(\beta)}-s_{0 i}^{(\alpha)}$. Для установления зависимости молярного объема $v_{0 i}^{(\alpha)}$ от $r$ можно воспользоваться разложением в ряд молярной плотности $\rho_{0 i}^{(\alpha)}=1 / v_{0 i}^{(\alpha)}$ по кривизне $R=1 / r$. Если ограничиться в этом разложении первым членом по $R$ (что справедливо для малой кривизны), будем иметь $[19,20]$

$$
v_{0 i}^{(\alpha)}(r) \approx v_{0 i \infty}^{(\alpha)} /\left(1+\frac{2 \sigma_{0 i \infty}^{(\alpha)} \chi_{0 i \infty}^{(\alpha)}}{r}\right)
$$

где $\sigma_{0 i \infty}^{(\alpha)}$ и $\chi_{0 i \infty}^{(\alpha)}-$ поверхностное натяжение и изотермическая сжимаемость $i$-компонента в фазе $\alpha$ при $r=\infty$. При проведении численных расчетов использовалось приближенное выражение $\sigma_{0 i \infty}^{(\alpha)} \chi_{0 i \infty}^{(\alpha)} \approx \frac{3}{2} \frac{\alpha_{0 i \infty}^{(\alpha)} T_{0 i \infty}\left(v_{0 i \infty}^{(\alpha)}\right)^{1 / 3}}{N_{0}^{1 / 3}}[21]$, где $\alpha_{0 i \infty}^{(\alpha)}$ - изобарический коэффициент теплового линейного расширения, $N_{0}$ - число Авогадро.

Молярная поверхность может быть выражена в виде

$$
\omega_{0 i}(r)=\frac{N_{0}^{1 / 3}}{n}\left[v_{0 i}^{(\sigma)}(r)\right]^{2 / 3},
$$

где $n$ - число монослоев в переходном слое. Величину молярного объема в межфазном слое можно приближенно находить, сохраняя для искривленной поверхности по форме соотношение, использованное для макроскопического случая [1].

Размерная зависимость скачка молярной энтропии при фазовом переходе $\alpha \rightarrow \beta$ приближенно находилась из (4) и (6) с использованием в целях упрощения формулы Толмена для $\sigma_{0 i}(r)$ и формулы Томсона для $T_{0 i}(r)$

$$
\begin{aligned}
s_{0 i}^{(\beta)}(r) & -s_{0 i}^{(\alpha)}(r) \\
& \approx \frac{s_{0 i \infty}^{(\beta)}-s_{0 i \infty}^{(\alpha)}}{\left(1+\frac{2 \bar{\delta}_{0 i}}{r}\right)\left(1+\frac{2 \sigma_{0 i \infty}^{(\alpha)} \chi_{0 i \infty}^{(\alpha)}}{r}\right)}\left(1-\frac{2 \delta_{0 i}^{*}}{r}\right) .
\end{aligned}
$$

В принципе можно использовать более общие выражения для $\sigma_{0 i}$ и $T_{0 i}$, приведенные выше (соответственно (27) и (28)).

3.3. Размерные зависимости параметра квазихимического взаимодействия в фазах и расчет параметра Толмена. Для приближенной оценки влияния размера на параметр квазихимического взаимодействия в той или иной фазе воспользуемся выражением $Q=\bar{\xi} z$, где $\bar{\xi}=N_{0}\left(2 \varepsilon_{i j}-\varepsilon_{i i}-\varepsilon_{j j}\right) / 2$. Полагая $\bar{\xi}$ не зависящим от размера, можно записать $Q(r) \approx z(r) Q_{\infty} / z_{\infty}$. В термодинамических расчетах поверхностного натяжения бинарных растворов (см., например, [16]) аналогичный прием часто используется для нахождения энергии смешения в поверхностном слое. Воспользуемся выражением для размерной зависимости координационного числа, полученным в рабо- 
те [22], и запишем $Q(r)$ в виде

$$
Q(r) \approx Q_{\infty}\left[1-\frac{3}{4}\left(\frac{r_{0}}{r}\right)+\frac{1}{16}\left(\frac{r_{0}}{r}\right)^{3}\right],
$$

где $r_{0}-$ расстояние между ближайшими частицами.

Расчеты, проведенные в $[23,24]$, показывают, что в случае границы твердое тело-жидкость в однокомпонентной системе для межфазного натяжения получаются значения, удовлетворительно согласующиеся с наиболее надежными данными для большого числа металлов, при условии $\delta_{0 i}^{(\alpha \beta)} \approx \delta_{0 i}^{(\alpha \gamma)}+\delta_{0 i}^{(\beta \gamma)}$, где $\delta_{0 i}^{(\alpha \gamma)}$ и $\delta_{0 i}^{(\beta \gamma)}-$ параметр Толмена для фаз $\alpha$ и $\beta$ соответственно на границе с паром $\left(\gamma-\right.$ пар). Значения $\delta_{0 i}^{(\alpha \gamma)}$ и $\delta_{0 i}^{(\beta \gamma)}$, в свою очередь, можно находить по одной из известных методик (см., например, $[25,26,27])$. В наших расчетах они рассчитывались в соответствии с [25] для основных кристаллических структур.

\section{4. Результаты расчетов и их краткое обсуждение}

Для апробации полученных соотношений по нахождению состава сосуществующих фаз с учетом наноразмерных эффектов проведем расчет для бинарной системы Mo-Ru. При проведении расчетов вычисляются также значения межфазного натяжения и концентрации компонентов на границе наночастицы и матрицы в зависимости от температуры.

В системе $\mathrm{Mo}-\mathrm{Ru}$ сосуществуют фазы: твердый раствор с ОЦК-решеткой и жидкость $(\beta-\mathrm{L})$, твердый раствор с ГПУ-решеткой и жидкость $(\varepsilon-\mathrm{L})$, а также твердый раствор с ОЦК-решеткой и твердый раствор с ГПУ-решеткой $(\beta-\varepsilon)$.

4.1. Входные данные для расчетов. Для молярных объемов компонентов в стабильных модификациях при температурах плавления $(r=\infty)$ были приняты значения $v_{\mathrm{Mo} \infty}^{(\beta)}=9.4 \mathrm{~cm}^{3} / \mathrm{mol}$ [28], $v_{\mathrm{Ru}}^{(\varepsilon)}=8.4 \mathrm{~cm}^{3} / \mathrm{mol}$ [28]. Объемы в случаях нестабильных модификаций (Мо с ГПУ-структурой, $\mathrm{Ru}$ c ОЦК-структурой) определялись с использованием коэффициентов упаковок для ОЦК- $(\eta=0.68)$ и ГПУ $(\eta=0.74)$-структур [29]. При приведении молярных объемов к соответствующим температурам принимались значения изобарических коэффициентов теплового объемного расширения, равные $\alpha_{V}^{\mathrm{Mo}}=50 \cdot 10^{-6} \mathrm{~K}^{-1}[29,30]$ и $\alpha_{V}^{\mathrm{Ru}}=50 \cdot 10^{-6} \mathrm{~K}^{-1}[29,30]$. Значения $r_{0}$ находились с использованием молярных объемов.

Межфазное натяжение на плоской границе макроскопических фаз твердое тело-жидкость в однокомпонентных системах может быть рассчитано с использованием ряда соотношений (см., например, $[16,23,28,31,32])$. После анализа результатов таких расчетов для межфазного натяжения и его температурного коэффициента нами использованы соотношения, предложенные в [28] (как наиболее удобные). Результаты вычислений $\sigma_{0 i \infty}^{(S L)}$ по методике [23] также хорошо согласуются с имею- щимися экспериментальными данными (в работе [23] рассчитаны значения межфазного натяжения на границе твердое-жидкость для 50 металлов).

Для характеристик фазового равновесия $\beta$-L в системе $\mathrm{Mo}-\mathrm{Ru}$ использовались значения: $T_{01 \infty}=2900 \mathrm{~K}, T_{02 \infty}=$ $=1420 \mathrm{~K}, s_{01 \infty}^{(\mathrm{L})}-s_{01 \infty}^{(\beta)}=8.368 \cdot 10^{7} \mathrm{erg} / \mathrm{mol}, s_{02 \infty}^{(\mathrm{L})}-s_{02 \infty}^{(\beta)}=$ $=11.715 \cdot 10^{7} \mathrm{erg} / \mathrm{mol}$ (все из [11]).

Для молибдена в $\beta$-модификации при температуре плавления получено $\sigma_{\mathrm{Mo} \infty}=464 \mathrm{~mJ} / \mathrm{m}^{2}$, $\frac{d \sigma_{\text {Мо }}}{d T}=0.0303 \mathrm{~mJ} /\left(\mathrm{m}^{2} \cdot \mathrm{K}\right) . \quad$ При нахождении аналогичных величин для рутения в метастабильной модификации $(\beta)$ использовалось соотношение между энтальпиями фазовых превращений $\beta-\mathrm{L}$ и $\varepsilon-\mathrm{L}, \quad$ приведенные в $[11]$, а также коэффициенты упаковки для $\beta$ и $\varepsilon$ структур [33]. Найденные таким образом величины составили $\sigma_{\mathrm{Ru} \infty}=289 \mathrm{~mJ} / \mathrm{m}^{2}$ и $\frac{d \sigma_{\mathrm{Ru} \infty}}{d T}=0.0476 \mathrm{~mJ} /\left(\mathrm{m}^{2} \cdot \mathrm{K}\right)$ при $T_{\mathrm{Ru} \infty}^{(\beta \mathrm{L})}=1420 \mathrm{~K}$.

Отметим, что приведенные выше положительные значения температурных коэффициентов межфазного натяжения на границе твердое тело-жидкость не должны вызывать возражений, так как они не противоречат термодинамике поверхности (см. формулу (12)). Аналогичные результаты (т.е. $\left.d \sigma_{0 i}^{\mathrm{SL}}>0\right)$ получены в рамках теории термодинамического подобия при фазовых переходах кристалл-жидкость [34]. При высоких температурах эта производная, как и на границе жидкость-пар [1], отрицательна вследствие возрастания амплитуды атомных колебаний с ростом температуры. Вычисленные нами значения параметpa Толмена по методике [25] и мольных площадей с использованием соотношения при температуре равновесия твердое тело $(\beta)$-жидкость соответственно равны $\delta_{01}=0.0276 \cdot 10^{-7} \mathrm{~cm}, \delta_{02}=0.0276 \cdot 10^{-7} \mathrm{~cm}$, $\omega_{01 \infty}=19.618 \cdot 10^{7} \mathrm{~cm}^{2}, \omega_{02 \infty}=19.888 \cdot 10^{7} \mathrm{~cm}^{2}$.

Для случая равновесия $\varepsilon$-L были приняты значения: $\quad T_{01 \infty}=1900 \mathrm{~K}, \quad T_{02 \infty}=2550 \mathrm{~K}$, $s_{01 \infty}^{(\mathrm{L})}-s_{01 \infty}^{(\varepsilon)}=8.368 \cdot 10^{7} \mathrm{erg} /(\mathrm{mol} \cdot \mathrm{K}), \quad s_{02 \infty}^{(\mathrm{L})}-s_{02 \infty}^{(\varepsilon)}=$ $=8.368 \cdot 10^{7} \mathrm{erg} /(\mathrm{mol} \cdot \mathrm{K}) \quad($ все из $[11]), \quad \sigma_{01 \infty}=$ $=324 \mathrm{~mJ} / \mathrm{m}^{2}, \frac{d \sigma_{01 \infty}}{d T}=0.0378 \mathrm{~mJ} /\left(\mathrm{m}^{2} \cdot \mathrm{K}\right), \sigma_{02 \infty}=389 \mathrm{~mJ} / \mathrm{m}^{2}$, $\frac{d \sigma_{02 \infty}}{d T}=0.0293 \mathrm{~mJ} /\left(\mathrm{m}^{2} \cdot \mathrm{K}\right), \quad \omega_{01 \infty}^{(\varepsilon \mathrm{L})}=18.328 \cdot 10^{7} \mathrm{~cm}^{2}$, $\omega_{02 \infty}=18.199 \cdot 10^{7} \mathrm{~cm}^{2}, \quad \delta_{01}=0.0875 \cdot 10^{-7} \mathrm{~cm}, \quad \delta_{02}=$ $=0.0847 \cdot 10^{-7} \mathrm{~cm}$.

Для характеристики фазового равновесия $\beta$ - $\varepsilon$ использованы значения: $\quad \omega_{01 \infty}=23.628 \cdot 10^{7} \mathrm{~cm}^{2}$, $\omega_{02 \infty}=21.729 \cdot 10^{7} \mathrm{~cm}^{2}, \delta_{01}=0.0347 \cdot 10^{-7} \mathrm{~cm}, \delta_{01}^{(\varepsilon \beta)}=$ $=0.0344 \cdot 10^{-7} \mathrm{~cm}$,

Межфазное натяжение на границе полиморфных фаз для компонентов рассчитывалось нами в соответствии с рекомендациями [28]. В результате были получены следующие значения для случая равновесия фаз $\alpha$ и $\beta$ :

$$
\begin{aligned}
& \sigma_{01 \infty}=874 \mathrm{~mJ} / \mathrm{m}^{2}, \quad \frac{d \sigma_{01 \infty}}{d T}=0.0612 \mathrm{~mJ} /\left(\mathrm{m}^{2} \cdot \mathrm{K}\right), \\
& \sigma_{02 \infty}=746 \mathrm{~mJ} / \mathrm{m}^{2}, \quad \frac{d \sigma_{02 \infty}^{(\varepsilon \beta)}}{d T}=0.0588 \mathrm{~mJ} /\left(\mathrm{m}^{2} \cdot \mathrm{K}\right)
\end{aligned}
$$

при $T=2000 \mathrm{~K}$. 
Для параметров квазихимического взаимодействия в сосуществующих фазах $(\mathrm{L}, \beta, \varepsilon)$ использовались различные значения, в том числе приведенные в [11], которые имеют отрицательные значения $Q_{\infty}^{(\mathrm{L})}=-23690 \cdot 10^{7} \mathrm{erg} / \mathrm{mol}, Q_{\infty}^{(\beta)}=-23937 \cdot 10^{7} \mathrm{erg} / \mathrm{mol}$, $Q_{\infty}^{(\varepsilon)}=-30967 \cdot 10^{7} \mathrm{erg} / \mathrm{mol}$. Аналогичный параметр для межфазного слоя приближенно может быть рассчитан с учетом числа атомных связей в пограничном слое, замыкающихся на прилегающие фазы [33]. Отметим, что находимые таким образом (в результате достаточно громоздких вычислений) значения $Q_{\infty}^{(\sigma)}$ для соответствующих межфазных границ в наших расчетах занимали промежуточное (близкое к среднему) положение по сравнению со значениями в сосуществующих фазах.

4.2. Результаты численных расчетов и их краткое обсуждение. На рис. 1,2 и в табл. 1 и 2 приведены результаты наших расчетов. Аналогичные данные получены нами для границ $(\varepsilon-\mathrm{L})$ и растворимости рутения в твердой фазе $\varepsilon$ (ГПУ).

На первом этапе расчеты проводились для случая, когда химические потенциалы компонентов во всех фазах в бинарной системе $\mathrm{Mo}-\mathrm{Ru}$ пропорциональны логарифмам концентраций $\left(\mu_{i} \sim \ln x_{i}\right)$. В этом случае удается получить в аналитическом виде выражения для всех неизвестных величин $\left(x_{i}^{(\alpha)}, x_{i}^{(\beta)}, x_{i}^{(\sigma)}, \sigma\right)$ в зависимости от радиуса поверхности натяжения.

Результаты наших расчетов состава сосуществующих макроскопических фаз $(r=\infty)$ и в двухфазной системе, содержащей наночастицы сферической формы с радиусом поверхности натяжения $r=5 \mathrm{~nm}$, в зависимости от температуры приведены на рис. 1. При этом вычисления проведены для случаев равновесия твердой фазы с ОЦК-решеткой (фаза $\beta$ ) и жидкости, твердой фазы с ГПУ-решеткой (фаза $\varepsilon$ ) и жидкости, а также двух твердых фаз с ОЦК- и ГПУ-структурами $(\beta-\varepsilon)$. Этих данных достаточно для построения фазовой диаграммы состояния макроскопических фаз и с учетом

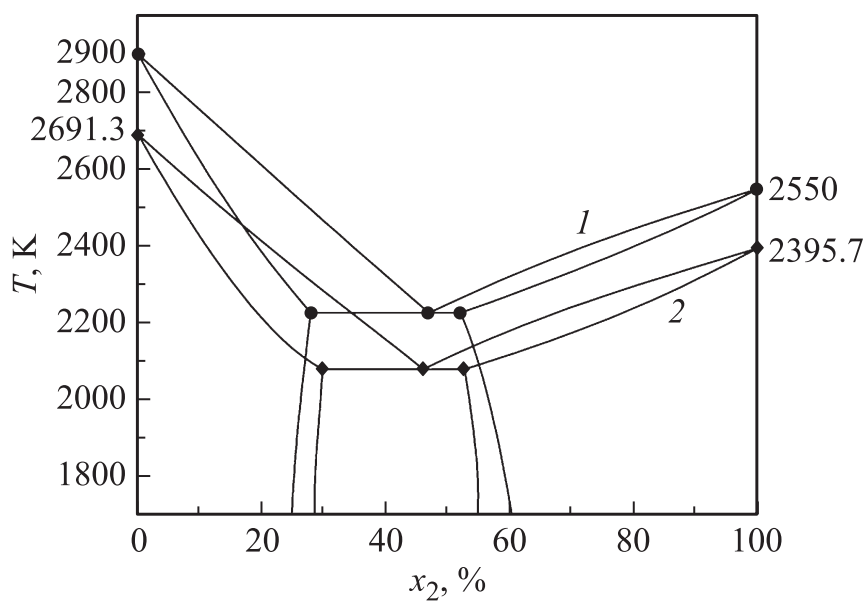

Рис. 1. Диаграммы состояния для системы Мo-Ru $1-\infty$, $2-r=5 \mathrm{~nm}$ (расчет).

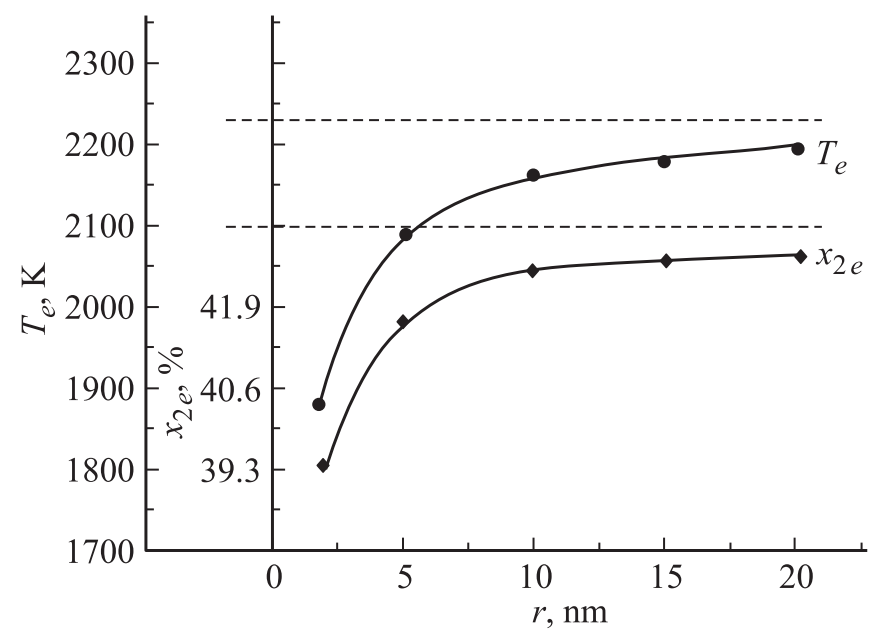

Рис. 2. Размерная зависимость координат эвтектической точки на диаграмме состояния в системе $\mathrm{Mo}-\mathrm{Ru}$.

наноразмерных эффектов. При $r=\infty$ результаты расчетов, несмотря на принятые допущения, достаточно хорошо согласуются с экспериментальными данными. Так, по результатам вычислений для случая, когда $\mu_{i} \sim \ln x_{i}$, диаграмма состояния относится к эвтектическому типу с твердыми растворами, что совпадает с экспериментальными данными [35-37]. Температура и состав в эвтектической точке, вычисленные нами в этом приближении, составляют соответственно $T_{e}=2228 \mathrm{~K}$, $x_{\mathrm{Ru} e}^{(\mathrm{L})} \approx 0.470$ и они близки к экспериментальным данным в [35] $\left(T_{e}=2228 \mathrm{~K}, x_{\mathrm{Ru} e}^{(\mathrm{L})} \approx 0.420\right)$ и в [37] $\left(T_{e}=2218 \mathrm{~K}\right.$, $\left.x_{\mathrm{Ru} e}^{(\mathrm{L})} \approx 0.416\right)$.

Эксперименты [35-37] показывают, что в бинарной системе Mo-Ru на всем температурном интервале ниже эвтектической температуры для твердых растворов на основе молибдена $d x_{\mathrm{Rue}}^{(\beta)} / d T>0$. По нашим расчетам, эта производная имеет такой знак при $T<1400 \mathrm{~K}$ и меняет знак $\left(d x_{\mathrm{Ru} \infty}^{(\beta)} / d T<0\right)$ при переходе к более высоким температурам. Эта связано, видимо, с тем, что в области температур от 1416 до $2228 \mathrm{~K}$ в этой системе существует, как показывают эксперименты $[35,36]$, промежуточная фаза ( $\sigma$-фаза) $\mathrm{Mo}_{5} \mathrm{Ru}_{3}$, которая нами не принималась во внимание при проведении расчетов. При температурах ниже температуры стабильности $\sigma$-фазы $(T<1416 \mathrm{~K}[35])$ наши расчетные данные показывают, возрастание растворимости рутения в молибдене в твердом состоянии с ростом температуры. Растворимость молибдена в рутении по нашим расчетам во всем температурном интервале в твердом состоянии $\left(T<T_{e}\right)$ растет с температурой, что соответствует экспериментам [35-37].

По найденным значениям координат эвтектической точки на диаграмме состояния можно также (второй вариант) построить кривые растворимости в твердом состоянии. Для этого из условий равновесия фаз $\beta$ и 
Таблица 1. Состав сосуществующих фаз $(\beta-\mathrm{L})$ в зависимости от температуры в системе Мо-Ru в макроскопическом случае $(r=\infty)$ и с учетом наноразмерных $(r \neq \infty)$ эффектов (расчет при $Q_{\infty}^{(\mathrm{L})}=-23.69 \mathrm{~kJ} / \mathrm{mol}, Q_{\infty}^{(\beta)}=-23.937 \mathrm{~kJ} / \mathrm{mol}$ )

\begin{tabular}{|c|c|c|c|c|c|c|c|c|c|c|}
\hline \multirow{3}{*}{$T, \mathrm{~K}$} & \multicolumn{5}{|c|}{$x_{2}^{(\beta)}, \%$} & \multicolumn{5}{|c|}{$x_{2}^{(\mathrm{L})}, \%$} \\
\hline & \multicolumn{10}{|c|}{$r, \mathrm{~nm}$} \\
\hline & $\infty$ & 15 & 10 & 5 & 2 & $\infty$ & 15 & 10 & 5 & 2 \\
\hline 2900 & 0 & & & & & 0 & & & & \\
\hline 2830 & 2.5 & 0 & & & & 4.7 & 0 & & & \\
\hline 2800 & 3.6 & 1.1 & & & & 6.7 & 2.1 & & & \\
\hline 2796 & 3.7 & 1.22 & 0 & & & 6.9 & 2.4 & 0 & & \\
\hline 2700 & 7.6 & 4.9 & 3.6 & & & 13.2 & 8.9 & 6.6 & & \\
\hline 2691 & 8.0 & 5.3 & 3.9 & 0 & & 13.7 & 9.5 & 7.2 & 0 & \\
\hline 2600 & 12.0 & 9.2 & 7.8 & 3.6 & & 19.6 & 15.5 & 13.3 & 6.6 & \\
\hline 2500 & 16.8 & 13.8 & 12.4 & 8.0 & & 25.9 & 22.0 & 20.0 & 13.6 & \\
\hline 2400 & 22.0 & 19.0 & 17.4 & 12.8 & & 32.4 & 28.6 & 26.6 & 20.5 & \\
\hline 2378 & 23.2 & 20.1 & 18.6 & 14.0 & 0 & 33.8 & 30.1 & 28.1 & 22.0 & 0 \\
\hline 2300 & 27.5 & 24.5 & 22.9 & 18.2 & 3.5 & 38.9 & 35.2 & 33.1 & 27.4 & 6.4 \\
\hline 2230 & 28.1 & 28.6 & 27.0 & 22.2 & 7.1 & 43.2 & 40.0 & 38.1 & 32.4 & 12.0 \\
\hline 2200 & & 30.4 & 28.8 & 24.0 & 8.7 & & 42.0 & 40.2 & 34.4 & 14.4 \\
\hline 2189 & & 31.1 & 29.5 & 24.7 & 9.31 & & 42.8 & 40.9 & 35.3 & 15.3 \\
\hline 2165 & & & 31.0 & 26.2 & 10.7 & & & 42.5 & 36.7 & 17.2 \\
\hline 2100 & & & & 30.3 & 14.5 & & & & 41.6 & 22.4 \\
\hline 2097 & & & & 30.5 & 14.7 & & & & 41.9 & 22.6 \\
\hline 2000 & & & & & 21.0 & & & & & 30.4 \\
\hline 1890 & & & & & 28.8 & & & & & 39.4 \\
\hline
\end{tabular}

Таблица 2. Растворимость рутения в твердой фазе $\beta$ (ОЦК) при разных температурах в системе Мо-Ru в макроскопическом случае $(r=\infty)$ и с учетом наноразмерных эффектов (расчет по формулам (33) и (34))

\begin{tabular}{|c|c|c|c|c|c|c|c|c|c|c|}
\hline \multirow{4}{*}{$T, \mathrm{~K}$} & \multicolumn{5}{|c|}{$Q_{\infty}^{(\beta)}=Q_{\infty}^{(\varepsilon)}=0$} & \multicolumn{5}{|c|}{$\begin{array}{c}Q_{\infty}^{(\beta)}=-23.937 \mathrm{~kJ} / \mathrm{mol}, \\
Q_{\infty}^{(\varepsilon)}=-23.0 \mathrm{~kJ} / \mathrm{mol}\end{array}$} \\
\hline & \multicolumn{5}{|c|}{$x_{2}^{(\beta)}, \%$} & \multicolumn{5}{|c|}{$x_{2}^{(\beta)}, \%$} \\
\hline & \multicolumn{10}{|c|}{ Радиус наночастицы $r, \mathrm{~nm}$} \\
\hline & $\infty$ & 15 & 10 & 5 & 2 & $\infty$ & 15 & 10 & 5 & 2 \\
\hline 600 & 11.6 & 11.9 & 12.1 & 12.8 & 15.3 & 20.2 & 22.9 & 23.0 & 22.9 & 22.6 \\
\hline 700 & 13.8 & 14.0 & 14.3 & 14.9 & 17.3 & 21.0 & 23.7 & 23.8 & 23.7 & 23.3 \\
\hline 800 & 15.7 & 15.9 & 16.1 & 16.7 & 19.0 & 21.7 & 24.4 & 24.5 & 24.4 & 23.9 \\
\hline 900 & 17.3 & 17.5 & 17.7 & 18.3 & 20.3 & 22.4 & 25.1 & 25.1 & 25.0 & 22.6 \\
\hline 1000 & 18.7 & 18.9 & 19.1 & 19.6 & 21.5 & 23.0 & 25.7 & 25.8 & 25.6 & 23.3 \\
\hline 1100 & 20.0 & 20.1 & 20.3 & 20.8 & 22.5 & 23.6 & 26.3 & 26.3 & 26.2 & 23.9 \\
\hline 1200 & 22.1 & 21.2 & 21.4 & 21.9 & 23.4 & 24.1 & 26.9 & 25.9 & 26.7 & 24.5 \\
\hline 1300 & 22.1 & 22.2 & 22.4 & 22.8 & 24.2 & 24.6 & 27.4 & 27.4 & 27.2 & 25.1 \\
\hline 1400 & 23.0 & 23.1 & 23.3 & 23.7 & 24.9 & 25.1 & 27.9 & 27.9 & 27.7 & 25.6 \\
\hline 1500 & 23.8 & 23.9 & 24.1 & 24.4 & 25.5 & 25.5 & 28.4 & 28.4 & 28.1 & 26.1 \\
\hline 1600 & 24.6 & 24.7 & 24.8 & 25.1 & 26.1 & 26.0 & 28.8 & 28.8 & 28.6 & 26.6 \\
\hline 1700 & 25.3 & 25.3 & 25.5 & 25.9 & 26.6 & 26.3 & 29.2 & 29.2 & 29.0 & 27.0 \\
\hline 1800 & 25.9 & 26.0 & 26.1 & 26.1 & 26.4 & 27.1 & 26.7 & 29.7 & 29.7 & 27.4 \\
\hline 1890 & 26.5 & 26.5 & 26.6 & 26.9 & 27.5 & 27.0 & 30.0 & 30.0 & 29.7 & 28.1 \\
\hline 2000 & 27.1 & 27.1 & 27.2 & 27.4 & 28.0 & 27.4 & 30.4 & 30.4 & 30.2 & 28.7 \\
\hline 2097 & 27.6 & 27.6 & 27.7 & 27.9 & 28.3 & 27.7 & 30.8 & 30.7 & 30.5 & 28.8 \\
\hline 2189 & 28.0 & 28.0 & 28.0 & 28.3 & 28.7 & 28.0 & 31.1 & 31.1 & 30.8 & 29.6 \\
\hline 2230 & 28.2 & 28.2 & 28.3 & 28.5 & 28.8 & 28.1 & 31.2 & 31.2 & 31.0 & 29.7 \\
\hline
\end{tabular}


$\varepsilon$ можно найти следующие соотношения

$$
\begin{gathered}
\left(s_{01 \infty}^{(\beta)}-s_{01 \infty}^{(\varepsilon)}\right)\left(T-T_{e \infty}\right)=R T \ln \frac{1-x_{2}^{(\beta)}}{1-x_{2}^{(\varepsilon)}}-R T \ln \frac{1-x_{2 e}^{(\beta)}}{1-x_{2 e}^{(\varepsilon)}} \\
+Q_{\infty}^{(\beta)}\left[\left(x_{2}^{(\beta)}\right)^{2}-\left(x_{2 e}^{(\beta)}\right)^{2}\right]-Q_{\infty}^{(\varepsilon)}\left[\left(x_{2}^{(\varepsilon)}\right)^{2}-\left(x_{2 e}^{(\varepsilon)}\right)^{2}\right], \\
\left(s_{02 \infty}^{(\beta)}-s_{02 \infty}^{(\varepsilon)}\right)\left(T-T_{e \infty}\right)=R T \ln \frac{x_{2}^{(\beta)}}{x_{2}^{(\varepsilon)}}-R T \ln \frac{x_{2 e}^{(\beta)}}{x_{2 e}^{(\varepsilon)}} \\
+Q_{\infty}^{(\beta)}\left[\left(1-x_{2}^{(\beta)}\right)^{2}-\left(1-x_{2 e}^{(\beta)}\right)^{2}\right] \\
-Q_{\infty}^{(\varepsilon)}\left[\left(1-x_{2}^{(\varepsilon)}\right)^{2}-\left(1-x_{2 e}^{(\varepsilon)}\right)^{2}\right],
\end{gathered}
$$

где $x_{2 e}^{(\beta)}$ и $x_{2 e}^{(\varepsilon)}-$ концентрации второго компонента при эвтектической температуре $T_{e}$ в фазах $\beta$ и $\varepsilon$ соответственно.

Разности энтропий ГПУ $(\varepsilon)$ - и ОЦК $(\beta)$-модификацией компонентов, входящие в (33) и (34), можно определить с использованием аппроксимации, предложенной в [11], для параметров стабильности металлов, которые равны $\Delta s_{01 \infty}^{(\beta \rightarrow \varepsilon)} \approx-0.38 \mathrm{cal} /(\mathrm{mol} \cdot \mathrm{deg})$ и $\Delta s_{02 \infty}^{(\beta \rightarrow \varepsilon)} \approx 0.80 \mathrm{cal} /(\mathrm{mol} \cdot \mathrm{deg})$. Результаты вычислений $x_{2 \infty}^{(\beta)}$ и $x_{2 \infty}^{(\varepsilon)}$ при $T<T_{e}$ по такой методике при $Q_{\infty}^{(\beta)}=Q_{\infty}^{(\varepsilon)}=0$ имеют меньшие значения по сравнению с аналогичными данными на рис. 1 и лучше согласуются с экспериментальными данными [35], а производные от этих концентраций по температуре имеют правильные знаки.

Учет межчастичных взаимодействий в рамках концепции регулярных растворов для макроскопических фаз (табл. 1,2) приводит к изменению положений линий ликвидуса и солидуса по сравнению со случаем идеальности в соответствии с закономерностями для фаз с отрицательным знаком параметра квазихимического взаимодействия в сосуществующих фазах. При этом принятые нами значения $Q_{\infty}^{(\mathrm{L})}, Q_{\infty}^{(\beta)}$ и $Q_{\infty}^{(\varepsilon)}$ (они сравнительно невелики по абсолютным значениям) приводят к уменьшению концентрации рутения и небольшому возрастанию температуры в эвтектической точке. Более заметные изменения имеют место по взаимной растворимости в твердом состоянии, где наблюдается увеличение растворимости рутения в молибдене и молибдена в рутении.

Расчеты по приведенным выше соотношениям с использованием указанных входных данных показывают, что при учете наноразмерных эффектов в данной системе сохраняется тип фазовой диаграммы состояния (эвтектической с ограниченной растворимостью в твердом состоянии) (рис. 1). При этом меняется положение линий ликвидуса и солидуса, а также растворимости в твердом состоянии по сравнению с макроскопическим случаем. Диаграмма состояния смещается вниз по температурной шкале и наблюдается расширение области существования жидкой фазы. Имеет место увеличение растворимости в твердом состоянии с ростом степени дисперсности.

Изменяются также координаты эвтектической точки (температура и состав) на фазовой диаграмме (рис. 2). Подобные результаты были получены и в ряде других работ (см., например, [38,6,39-41]). При этом, как отмечено в этих работах, может иметь место изменение типа диаграммы по сравнению с фазовой диаграммой для массивных образцов. Подобное качественное изменение в рассматриваемой нами системе Mo- Ru не наблюдалось, но имели место заметные изменения в составах сосуществующих фаз. Так, при диспергировании в жидком молибдене твердого рутения на частицы сферической формы с радиусом поверхности натяжения $r=5 \mathrm{~nm}$ при температуре $T=2300 \mathrm{~K}$ концентрация рутения в твердых наночастицах с ОЦК-решеткой по сравнению с массивными матрицами $\left(x_{2}^{(\beta)}<x_{2 \infty}^{(\beta)}\right)$ уменьшалась более чем в 1.5 раза. В то же время для случая $\varepsilon$-L равновесия при той же температуре концентрация рутения в твердой наночастице с ГПУ-решеткой с таким же радиусом кривизны возросла в 1.4 раза по сравнению с макроскопическим случаем $\left(x_{2}^{(\mathrm{L})}<x_{2 \infty}^{(\mathrm{L})}\right)$.

Аналогичное изменение концентрации имеет место и во второй фазе (жидкости).

В случае равновесия двух твердых фаз с ОЦК- и ГПУ-структурами в системе Мo-Ru с увеличением степени дисперсности имеет место сближение состава фаз, что в соответствии с [42], рассматривается в пользу флуктуационного характера зарождения частиц второй фазы.

Отметим, что указанные выше изменения концентраций в фазах, сосуществующих в равновесных условиях при разных радиусах поверхности натяжения, полученные в наших расчетах, зависят от знака и численных значений параметра Толмена на границе наночастицы и матрицы для каждого типа фазового равновесия $(\beta-\mathrm{L}$, $\varepsilon-\mathrm{L}, \beta-\varepsilon)$. Использованные нами значения этого параметра во всех случаях достаточно малы и составляют сотые доли нанометра. В подавляющем числе работ, посвященных расчету зависимости поверхностного натяжения от размера на границе с паром для $\delta_{0 i}$, используются значения, которые лежат в интервале от десятых долей до нескольких единиц нанометра $[38,42]$. По нашим оценкам [23] значения этого параметра для границы раздела двух конденсированных фаз должны быть выше по сравнению с границами этих фаз с паром. При принятии больших значений для параметра Толмена наши расчеты дают более значительные изменения в составах сосуществующих фаз в высокодисперсных системах.

\section{5. Заключение}

В рамках термодинамики фазовых равновесий в бинарных макро- и наносистемах при строгом учете поверхностных (межфазных) явлений получена замкнутая 
система уравнений в интегральной форме, позволяющая находить основные параметры термодинамического равновесия двухфазной системы с заданной степенью дисперсности (включая область наноразмеров). Новые соотношения, полученные для случая постоянства давления в матрице, в которой находятся дисперсные частицы сферической формы, позволяют учесть влияние наноразмерных эффектов на состав сосуществующих фаз и переходного слоя между ними, а также межфазное натяжение в зависимости от температуры. Описание фазовых равновесий проведено для случаев, когда химические потенциалы компонентов пропорциональны концентрациям и в рамках теории регулярных растворов. Переход на более сложные модели не представляет принципиальных трудностей и может быть осуществлен в рамках принятой схемы.

При устремлении радиуса кривизны (радиуса поверхности натяжения) к бесконечности эти соотношения позволяют строить фазовые диаграммы состояния в бинарных системах с макроскопическими фазами и находить межфазное натяжение и состав межфазного слоя на границах раздела для изобарического разреза зависимостей температуры, давления и концентраций $\left(T, P,\left\{x_{i}\right\}\right)$. При устремлении концентрации одного из компонентов к нулю полученные соотношения определяют размерные зависимости температуры равновесия, межфазного натяжения и скачка энтропии при фазовом переходе в однокомпонентных системах. Для случая малой кривизны из них следует известное соотношение Томсона для температуры плавления и формула Толмена для поверхностного (межфазного) натяжения. При соответствующих предположениях из них также следует формула Оствальда-Фройндлиха (ее называют также формулой Гиббса-Томсона) для растворимости малых частиц.

Численные расчеты проведены для системы $\mathrm{Mo}-\mathrm{Ru}$, где имеются фазовые равновесия с участием твердых растворов с ОЦК $(\beta)$ - и ГЦК $(\varepsilon)$-структурами и жидкости. При переходе к макроскопическим размерам, результаты расчетов согласуются с экспериментальной диаграммой состояния. Показано, что учет наноразмерных эффектов (изменения молярных объемов и площадей, скачка энтропий при фазовом переходе, температуры равновесия, межфазного натяжения и параметра квазихимического взаимодействия) приводит к смещению положений линий ликвидуса и солидуса, а также растворимости в твердом состоянии и координат эвтектической точки (состав, температура). Диаграмма состояния смещается вниз по температурной шкале с ростом степени диспергирования одной фазы в другой сосуществующей фазе (матрице).

\section{Список литературы}

[1] А.И. Русанов. Фазовые равновесия и поверхностные явления. Химия, Л. (1967). 388 с.

[2] В.К. Григорович. Жаропрочность и диаграмма состояния. Металлургия, М. (1969). 324 с.
[3] П.Е. Львов, В.В. Светухин, А.В. Обухов. ФТТ 53, 2, 394 (2011).

[4] А.Л. Удовский. Металлы 5, 121 (2011).

[5] А.Л. Удовский, Д.А. Васильев. Вестн. Новгородского гос. ун-та, 2, 73, 51, (2013).

[6] T. Tanaka. Mater. Sci. Forum 653, 55 (2010).

[7] А.И. Русанов. Лекции по термодинамике поверхностей. Лань, СПб. (2013). 240 с.

[8] А.Г. Морачевский. Физическая химия. Поверхностные явления и дисперсные системы. Лань, СПб (2015), 160 с.

[9] Г. Реза Вакили-Неджаад. Нанотермодинамика. В кн.: Нанонаука и нанотехнологии. EOLS, Магистр-Пресс, М. (2010). C. 78.

[10] А.А. Смирнов. Молекулярно-кинетическая теория металлов. Наука, М. (1966), 488 с.

[11] Л. Кауфман, Х. Бернстейн. Расчет диаграмм состояния с помощью ЭВМ. Мир, М. (1972), 326 с.

[12] 3.А. Шебзухов, М.А. Шебзухова, А.А. Шебзухов. Поверхность. Синхротронные и нейтронные исследования 11, 102 (2009).

[13] З.А. Шебзухов, М.А. Шебзухова, А.А. Шебзухов. Поверхность. Синхротронные и нейтронные исследования 12, 94 (2009).

[14] М.А. Шебзухова, А.А. Шебзухов. Изв. РАН. Сер. физ. 80, 6, 789 (2016).

[15] А.А. Шебзухов, А.М. Карачаев. Поверхность. Физика, химия, механика 58, 5, 58 (1984).

[16] С.И. Попель. Поверхностные явления в расплавах. Металлургия, М. (1994). 440 с

[17] 3.А. Шебзухов, М.А. Шебзухова, А.А. Шебзухов. Изв. Кабардино-Балкарского гос. ун-та им. Х.М. Бербекова 1, 17 (2010).

[18] М.А. Шебзухова, А.А. Шебзухов. Изв. РАН. Сер. физ. 76, 7, 863 (2012).

[19] А.И. Русанов. Коллоидный журн. XLIX, 5, 932 (1987).

[20] Zhu Ru-Zeng, Wang Xiao-Song. Chin. Phys. B 19, 7, 076801 (2010).

[21] В.В. Байдов, Л.Л. Кунин. В сб.: Поверхностные явления в расплавах и возникающих из них твердых фазах. / Под ред. С.Н. Задумкина. Кабардино-Балкарское книжн. изд-во, Нальчик (1965). С. 79.

[22] A.I. Frenkel, A. Yevick, Ch. Cooper, R. Vasic. Ann. Rev. Anal. Chem. 4, 23, (2011).

[23] М.А. Шебзухова, А.А. Шебзухов. ФТТ 54, 1, 173 (2012).

[24] М.А. Шебзухова, З.А. Шебзухов, А.А. Шебзухов. Изв. Кабардино-Балкарского гос. ун-та им. Х.М. Бербекова. 1, 3,83 (2011).

[25] М.А. Шебзухова, 3.А. Шебзухов, А.А. Шебзухов. Изв. РАН. Сер. физ. 74, 5, 751 (2010).

[26] Дж. Роулинсон, Б. Уидом. Молекулярная теория капиллярности. Мир, М. (1986). 375 с.

[27] W. Vogelsberger, G. Marx. Z. Phys. Chem. 257, 3, 580 (1976).

[28] Q. Jiang, H.M. Li. Surf. Sci. Rep. 63, 427 (2008).

[29] С.И. Новикова. Тепловое расширение твердых тел. Наука, M. (1974). 294 c.

[30] В.Е. Зиновьев, Е.Н. Келина, Л.И. Комарова. Плотность и термическое расширение переходных металлов при высоких температурах. Обзор по теплофизическим свойствам веществ. ИВТАН СССР, М. 3, 71 (1988). 100 с.

[31] С.Н. Задумкин. Физика металлов и металловедение 13, 24 (1962).

[32] L.A. Girifalco, R.I. Cood. J. Phys. Chem. 61, 904 (1957). 
[33] С.И. Попель, М.А. Спиридонов, Л.А. Жукова. Атомное упорядочение в расплавленных и аморфных металлах. УГТУ, Екатеринбург (1997). 384 с.

[34] В.П. Скрипов, М.3. Файзуллин. Фазовые переходы кристалл-жидкость-пар и термодинамическое подобие. Физматлит, М. (2003). 160 с.

[35] H. Kleykamp. J. Less-Common Met. 136, 271 (1988).

[36] R.Gürler. J. Alloys Comp. 285, 133 (1999).

[37] Диаграмма состояния двойных металлических систем / Под ред. Н.П. Лякишева. Машиностроение, М. (2001). Т. 3. Кн. 1.872 c.

[38] Н.Т. Гладких, С.В. Дукаров, А.П. Крышталь, В.И. Ларин, В.Н. Сухов, С.И. Богатыренко. В сб.: Поверхностные явления и фазовые превращения в конденсированных пленках / Под ред. Н.Т. Гладких. ХНУ им. В.Н. Каразина, Харьков (2004). 276 с.

[39] J. Sopousek, J. Vrestal, J. Pinkas, P. Broz, J. Bursik, A. Styskalik, D. Skoda, O. Zobac, J. Lee. Calphad 45, 33 (2014).

[40] A. Roshanghias, J. Vrestal, A. Yakymovgech, K. Richter, H. Ipser. Calphad 49, 101 (2015).

[41] S. Bajaj, M.G. Haverty, R. Arroyave, W.A. Goddard, S. Shankare. Nanoscale 7, 9868 (2015).

[42] П.Е. Львов, В.В. Светухин. Письма в ЖТФ 35, 22, 33 (2009). 